\title{
A THEOREM ON ACYCLICITY
}

\author{
BY A. D. WALLACE
}

Communicated by R. H. Bing, September 12, 1960

TheOREM. Let $X$ and $Y$ be compact Hausdorff spaces and let $M$ be a set-to-set function which assigns to each closed set $A$ in $X$ a closed set $M(A)$ in $Y$ and with the properties

(a) If $A_{1}$ and $A_{2}$ are closed subsets of $X$ then $M\left(A_{1} \cup A_{2}\right)=M\left(A_{1}\right)$ $\cup M\left(A_{2}\right)$,

(b) If $x \in X$ and if $V$ is an open set about $M(x)$ then there is an open set $U$ about $x$ with $M\left(U^{*}\right) \subset V$, the * denoting closure,

(c) If $A_{1}$ and $A_{2}$ are closed sets in $X$ then there is a closed set $A_{3}$ in $X$ such that $M\left(A_{1}\right) \cap M\left(A_{2}\right)=M\left(A_{3}\right)$,

(d) For any two closed sets $A_{1}$ and $A_{2}$ of $X$ the set $M\left(A_{1}\right) \cap M\left(A_{2}\right)$ is connected.

If also $Y_{0}$ is a closed subset of $Y$ and if $H^{p}\left(M(x), M(x) \cap Y_{0}\right)=0$ for each integer $p \geqq 1$ and each $x \in X$ then $H^{p}\left(M(A), M(A) \cap Y_{0}\right)=0$ for each closed subset $A$ of $X$ and each such integer $p$.

Although the theorem may, at first glimpse, seem closely related to results of Begle [2] and Vietoris [7], its relationship to propositions of Golab [3], Roberts [4], Rutt [5], and G. S. Young [8] is more immediate. It can be reformulated in several ways and the following corollary to one way of stating it will indicate its connection with a result of Golab's:

CoRollary. Let $M$ be an upper semicontinuous point-to-set function which assigns to each point $x$ of a simple closed curve $X$ a continuum containing $x$ which does not cut the plane and suppose, in addition, that all of the sets $M(x)$ have a point in common and that the intersection of any two of them is connected; then their union contains the cell bounded by $X$.

It should be observed that the hypotheses on $Y$ can be weakened and it is indeed enough to suppose that $Y$ is fully normal, or even less $[6 ; 9]$.

The condition (c), because of its unusual character, deserves a comment, which can best be put in this way. Suppose that $X$ and $Y$ are compact Hausdorff, that $R$ is a closed subset of $X \times X$ and that $M(A)$ is written for the second projection of $(A \times X) \cap R$; then (c) is satisfied if $R$ is both reflexive and transitive. When the remainder of the hypotheses are fulfilled the theorem can be applied to structs [10] as the following example of its use will show: 
COROLlARY. If $L$ is a monotone reflexive struct on the 2-sphere such that the set of minimal elements is connected and such that no set $L(x)$ cuts the 2-sphere then there is an element which is universally L-maximal.

For some years I have thought that there should be a version of the Morse minimax theory which involved structs, or at least continuous functions to partially ordered spaces. In a somewhat nebulous way the result of this announcement lends credence to this somewhat vague conjecture, though I would not be so bold as to suggest that this is entirely apparent to the casual reader.

The result has applications to the structure of semigroups, and in fact, is merely an abstraction of the arguments given in [11]. We state one result not given in that paper:

COROLlaRY. If $S$ is a compact connected regular $(x \in x S x$ for each $x \in S$ ) semigroup, if $I$ is a closed ideal and if $L$ is a closed left ideal then $H^{p}(S) \approx H^{p}(I \cup L)$ for each non-negative integer $p$ and if $S$ has a zero element it follows that $S$ is acyclic.

A recent result [1] of L. W. Anderson and L. E. Ward, Jr. is a consequence of this corollary and information concerning their result prompted a renewal of my interest in the acyclicity of semigroups.

I am greatly obliged to Miss Anne Lester and Professor R. J. Koch for their several fruitful comments and to the National Science Foundation for its support.

\section{BIBLIOGRAPHY}

1. L. W. Anderson and L. E. Ward, Jr., One dimensional topological semilattices, Illinois J. Math., to appear.

2. E. G. Begle, The Vietoris mapping theorem for bicompact space, Ann. of Math. vol. 41 (1950) pp. 534-543.

3. St. Golab, Un theorème sur le balayage, Fund. Math. vol. 12 (1928) pp. 4-19.

4. J. H. Roberts, Concerning collections of continua not all bounded, Amer. J. Math. vol. 52 (1930) pp. 551-562.

5. N. E. Rutt, On certain types of plane continua, Trans. Amer. Math. Soc. vol. 33 (1931) pp. 863-875.

6. Shozo Sakai, On the map excision theorem, Sci. Rep. Tokyo Bunrika Diagaku. Sect. A vol. 4 (1953) pp. 290-297.

7. L. Vietoris, Über den höheren Zusammenhang kompakter Räume, Math. Ann. vol. 97 (1927) pp. 454-472.

8. G. S. Young, A generalization of the Rutt-Roberts theorem, Proc. Amer. Math. Soc. vol. 4 (1951) pp. 586-588.

9. A. D. Wallace, The map excision theorem, Duke Math. J. vol. 20 (1952) pp. 177-182.

10. —- Struct ideals, Proc. Amer. Math. Soc. vol 6. (1955) pp. 634-638.

11. - Acyclicity of compact connected semigroups, Fund. Math., to appear.

The Tulane University of Louisiana 